\title{
Efficacy and Safety of Zofenopril Versus Ramipril in the Treatment of Myocardial Infarction and Heart Failure: A Review of the Published and Unpublished Data of the Randomized Double-Blind SMILE-4 Study
}

\author{
Claudio Borghi · Stefano Omboni - Salvatore Novo • Dragos Vinereanu • \\ Giuseppe Ambrosio · Ettore Ambrosioni
}

Received: February 14, 2018 / Published online: April 17, 2018

(C) The Author(s) 2018

\begin{abstract}
Zofenopril is a lipophilic, sulfhydryl groupcontaining angiotensin-converting enzyme (ACE)-inhibitor, characterized by wide tissue distribution, long duration of action, and pleiotropic effects on endothelial dysfunction. Its clinical efficacy and safety have been described in the four randomized controlled trials of the SMILE program, which globally enrolled more than 3600 patients in post-acute myocardial infarction (AMI) setting. The SMILE4 study specifically selected patients with left
\end{abstract}

Enhanced content To view enhanced content for this article go to https://doi.org/10.6084/m9.figshare.59522 89.

C. Borghi $(\bowtie) \cdot$ E. Ambrosioni

Unit of Internal Medicine, Policlinico S. Orsola,

University of Bologna, Bologna, Italy

e-mail: claudio.borghi@unibo.it

S. Omboni

Clinical Research Unit, Italian Institute of

Telemedicine, Varese, Italy

S. Novo

Division of Cardiology, Policlinico Giaccone,

University of Palermo, Palermo, Italy

D. Vinereanu

Cardiology, University and Emergency Hospital,

Bucharest, Romania

G. Ambrosio

Division of Cardiology, Ospedale S. Maria della

Misericordia, University of Perugia, Perugia, Italy ventricular dysfunction at admission, and compared the effects of zofenopril or ramipril in combination with acetylsalicylic acid (ASA). Zofenopril demonstrated its superiority over ramipril in reducing the combined occurrence of death or hospitalization for cardiovascular causes both in the overall population included in the original study and in subgroups of patients at highest risk, namely hypertensive and diabetic subjects. The effects of the early treatment with zofenopril were sustained over time, and, after 5 years of follow-up, zofenopril increased the survival likelihood and reduced the hospitalization rate. Compared to ramipril, zofenopril was cost-effective with a number to treat of 13 and an incremental cost-effectiveness ratio (ICER) of 2125.45 euros for any additional event prevented. Furthermore, in real-world settings, zofenopril decreased the risk of death in patients with heart failure, particularly in men, and in subjects older than 76 years or with ejection fraction lower than 54\%. These results support the early use of zofenopril immediately after AMI, even in the presence of comorbidities, and its maintenance over time to reduce the risk of heart failure.

Funding: Menarini International Operations Luxembourg S.A.

Keywords: Acute myocardial infarction; Angiotensin-converting enzyme inhibitors; 
Cardiology; Heart failure; Left ventricular dysfunction; Ramipril; Zofenopril

\section{INTRODUCTION}

Acute myocardial infarction (AMI) is often associated with activation of the circulating renin-angiotensin-aldosterone system (RAAS), which causes necrosis of cardiac myocytes and systemic vasoconstriction and affects left ventricular structure and function [1]. Therefore, current guidelines recommend angiotensinconverting enzyme (ACE)-inhibitors to all patients with ST-segment elevation AMI (STEMI) and an impaired left ventricular ejection fraction (LVEF $<40 \%$ ), or those who have experienced heart failure (HF) in the early phase $[2,3]$. A combination of ACE-inhibitors and acetylsalicylic acid (ASA) is often administered to improve outcomes in patients with left ventricular dysfunction (LVD), but potential negative interactions have questioned this approach.

This review will summarize the results from the SMILE-4 study, the clinical trial of SMILE program that specifically compared the effects of zofenopril or ramipril in combination with ASA in patients with LVD after AMI. Evidence from published data of the main study and subgroups, and unpublished data on the longterm effect of zofenopril and ramipril in the patients of the SMILE- 4 study, will be summarized and critically presented. This article is based on previously conducted studies and does not contain any new studies with human participants or animals performed by any of the authors.

\section{PHARMACOLOGICAL RATIONALE OF ZOFENOPRIL IN THE TREATMENT OF AMI AND HF}

Zofenopril is a sulfhydryl group-containing ACE-inhibitor, converted by esterases in the active metabolite zofenoprilat in both serum and tissues. The main feature of zofenopril is its high lipophilicity that favors a wide tissue distribution and a long duration of action $[4,5]$. The time course of tissue distribution is characteristic for each molecule and varies among ACE-inhibitors: all ACE-inhibitors achieve an early and complete inhibition of the ACE in the blood, but their activity in other tissues is mainly associated with a selective distribution rather than with a different inhibitory potency. For instance, in the aorta, the inhibitory activity of zofenopril persists for more than 4 days and, as well as in cardiac tissue, zofenopril produces a long-lasting inhibition [4]. The wide tissue distribution of zofenopril is even important for its pleiotropic effects on endothelial dysfunction, namely its anti-ischemic, anti-atherogenic, and pro-angiogenic properties [6].

In addition to high lipophilicity, the presence of the sulfhydryl group in the zofenopril structure provides vascular protection and antiatherogenic effects due to the ability to reduce the oxidative stress and activate the nitric oxide (NO) pathway [7]; therefore, its role on peripheral vascular function is independent of ACEinhibition [8].

Numerous evidence in animal models supports that the cardioprotective role of zofenopril after AMI is associated with anti-oxidant and anti-atherogenic properties. In Watanabe heritable hyperlipidemic rabbits, zofenopril reduced plasmatic low-density lipoprotein (LDL) oxidation, the number of macrophagesderived foam cells, and the wall-associated platelet deposition at the site of atherosclerotic lesions [9]. In rat models of ischemia and heart failure treated with zofenopril for 15 days (chronic model), zofenopril induced heat shock protein 70 expression and downregulated NO synthase 3 , with a heart-specific effect on gene expression which partially explained an increased resistance to ischemia [10]. Zofenopril significantly augmented both plasma and myocardial $\mathrm{H}_{2} \mathrm{~S}$ (sulfane sulfur) and $\mathrm{NO}$ levels in mice and plasma $\mathrm{H}_{2} \mathrm{~S}$ in pigs, substances that could scavenge radical oxygen substrates and prevent myocardial damages associated with the ischemia/reperfusion injury [11].

Therefore, both in vitro and in vivo data support the rationale use of zofenopril in patients after AMI. 


\section{CLINICAL EFFICACY OF ZOFENOPRIL IN AMI: THE SMILE PROGRAM}

Zofenopril has been tested in an extensive clinical program to assess its effects on reduction of morbidity and mortality in patients after AMI. The SMILE (Survival of Myocardial Infarction Long-term Evaluation) studies involved more than 3600 patients in different settings (Table 1$)$ : (1) early AMI $(<24 \mathrm{~h})$, not eligible for thrombolytic therapy because of late admission to the intensive care unit or with contraindication to systemic fibrinolysis (SMILE-1) [12]; (2) confirmed diagnosis of AMI and a prior thrombolytic treatment within $12 \mathrm{~h}$ from the onset of clinical symptoms (SMILE-2) [13]; (3) recent AMI (within $6 \pm 1$ weeks) with preserved LVEF ( $>40 \%$ ), treated with thrombolytic treatment and ACE-inhibitors (SMILE-3) [14]; and (4) early AMI (within $24 \mathrm{~h}$ ), either treated with thrombolysis or not, with primary percutaneous transluminal angioplasty (PCTA) or coronary artery by-pass graft, and with clinical and/or echocardiographic evidence of left ventricular dysfunction (SMILE-4) [15]. The program enrolled patients in almost all European Countries, thus providing data from subjects with different dietary and medical behaviours and in the context of different healthcare services; one-quarter of subjects enrolled were women.

In patients of the SMILE-1 Study with early AMI occurring within $24 \mathrm{~h}$, zofenopril reduced the risk of death or severe congestive heart failure by $34 \%(95 \% \mathrm{CI}, 8-54 \% ; p=0.018)$, and after 1 year of follow-up, reduced the risk of death by $29 \%$ (95\% CI, 6-51\%; $p=0.011)$, with a lower mortality rate than placebo $(10.0 \%$ vs. $14.1 \%)$ [12].

In the SMILE-2 Study, the safety of zofenopril treatment was compared to lisinopril in patients with different infarct sites who underwent thrombolytic therapy. Zofenopril, started within $24 \mathrm{~h}$ from symptoms onset, resulted as safe and well-tolerated and showed a rate of severe drug-related hypotension lower than lisinopril (6.7\% vs. $9.8 \%, p=0.048)[13]$.
In the SMILE-3 Study, electrocardiographic (ECG) abnormalities were evaluated after 6 months of treatment with zofenopril or placebo in patients with LVEF $>40 \%$. Zofenopril decreased the rate of ST-T depression during both the ambulatory ECG and the exercise test, reduced the incidence of anginal pain, significant ST depression, and major ventricular arrhythmias, thus supporting its cardioprotective role when ventricular function was preserved [14].

A pooled analysis of individual patient data from all the SMILE studies (the results of the SMILE-4 Study will be detailed and discussed in the next sections) further confirmed the efficacy of zofenopril in the post-AMI setting. Zofenopril reduced the risk of combined occurrence of death or hospitalization for $\mathrm{CV}$ causes, compared to placebo, and, at least in the long term, even compared to lisinopril and ramipril. The activity of zofenopril was evident immediately after starting the treatment and sustained over time: a risk reduction by $70 \%$ was achieved in the first 6 weeks, while an additional 30\% was reached at the end of follow-up [16].

\section{ZOFENOPRIL AND RAMIPRIL IN COMBINATION WITH ASA IN PATIENTS WITH LVD FOLLOWING AMI: THE SMILE-4 STUDY}

The SMILE-4 Study was designed to investigate the efficacy of zofenopril in combination with ASA [15]. A combination with ACE-inhibitors and ASA is frequently used to treat patients with LVD, but some concerns about possible negative interactions have questioned this approach. In the SMILE-4 Study, patients were randomized to receive zofenopril or ramipril plus ASA (100 mg once daily). As primary outcome, the 1-year combined occurrence of mortality or hospitalization for cardiovascular $(\mathrm{CV})$ disease was lower with zofenopril + ASA treatment than ramipril + ASA, with a reduction of risk by 30\% (OR 0.70, 95\% CI 0.51-0.96) (Fig. 1). Both systolic and diastolic blood pressure, median levels of NT-proBNP, improvement in LVEF, 


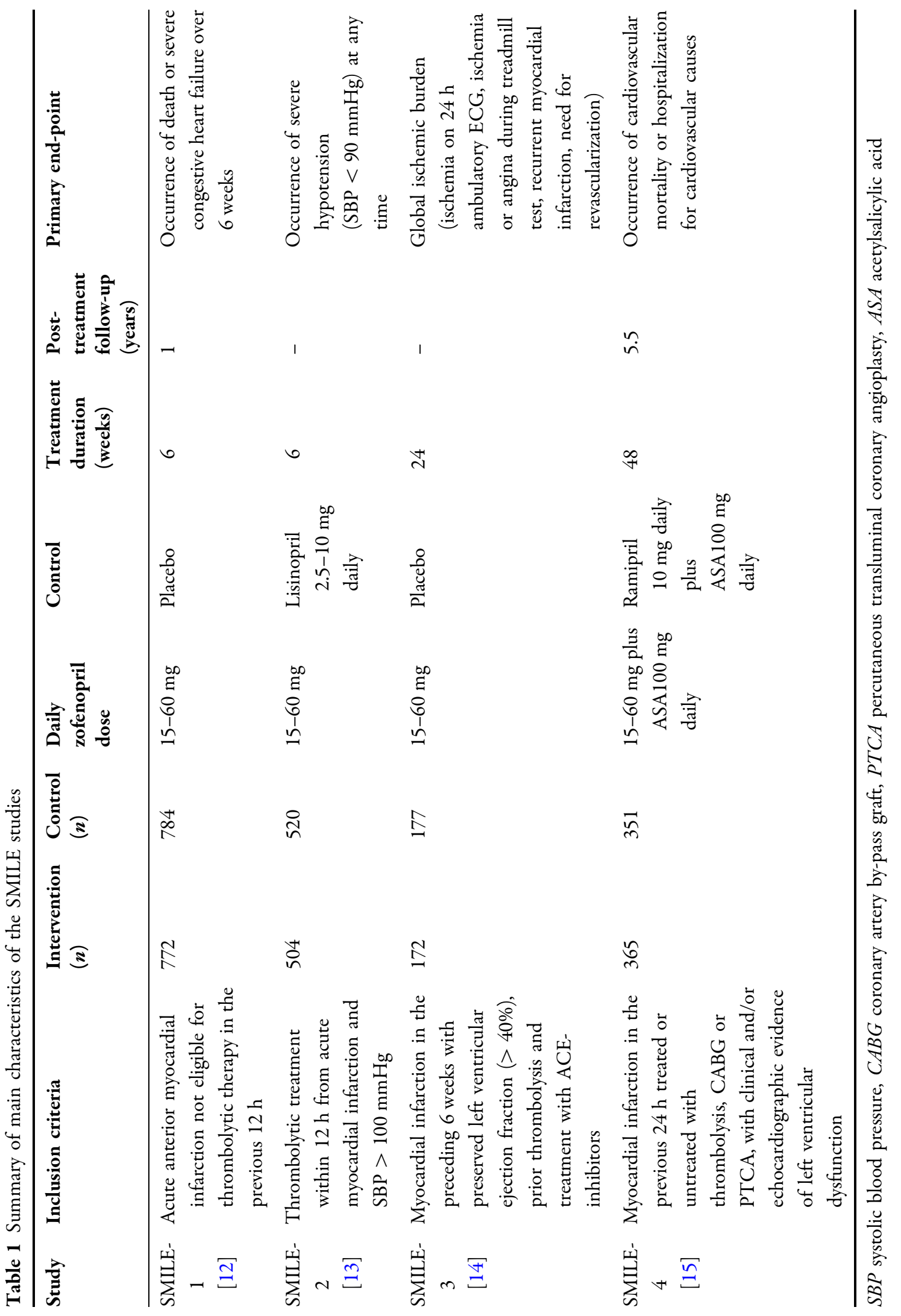




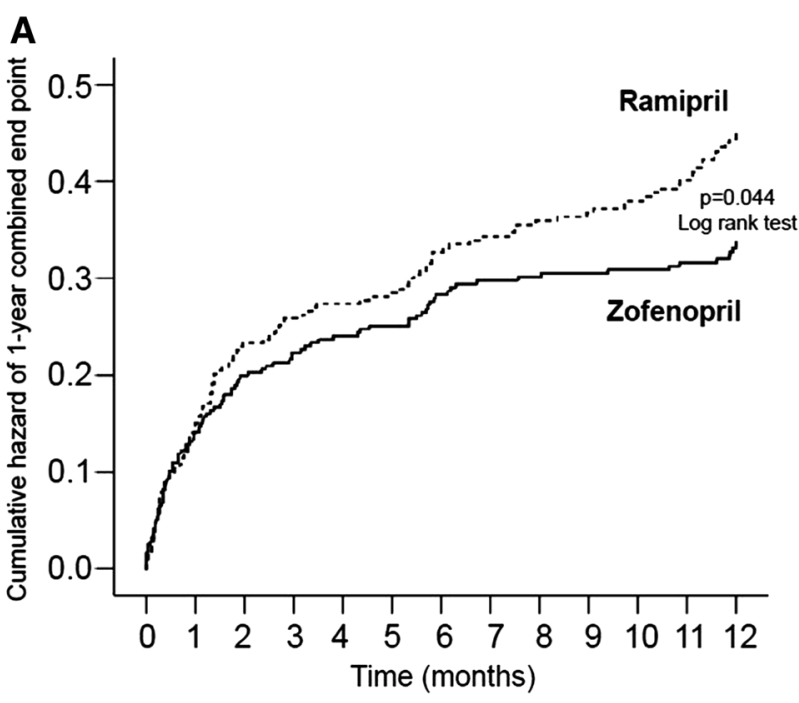

Number at risk

Zofenopril $\quad 365317298287281276267262261260258255247$

Ramipril $\quad 351305276269263260247243238236233228215$

Fig. 1 Cumulative incidence of the combined primary study endpoint ( $\mathrm{CV}$ mortality or hospitalization for $\mathrm{CV}$ causes, including congestive heart failure, acute myocardial infarction, angina or decline in left ventricular ejection fraction $>15 \%$ ) (a) and of hospitalization (b) during the 1 year of treatment

renal function deterioration, and non-CV adverse events were similar between treatments. Therefore, zofenopril + ASA was more efficient than ramipril + ASA in preventing the 1-year risk of death or hospitalization for CV causes [15]. The difference in clinical efficacy among ACE-inhibitors in association with ASA might be related to the presence of the sulfhydryl group-present in zofenopril but not in ramipril-which confers cardioprotective effects by mechanisms other than prostaglandin-mediated signalling. As a matter of fact, in the SMILE-1 study, the relative risk reduction of death or severe congestive heart failure after 6 weeks of treatment with zofenopril versus placebo was not significantly different $(p=0.364)$ in the presence of (RR $0.77,95 \% \mathrm{CI}$ $0.45-1.30 ; n=61$ ) or in the absence of (RR 0.55, 95\% CI $0.33-0.89 ; n=77$ ) concomitant ASA [12].

To further strengthen the results of the SMILE-4 Study, a propensity score analysis was performed to take into account some potential confounding factors related to population

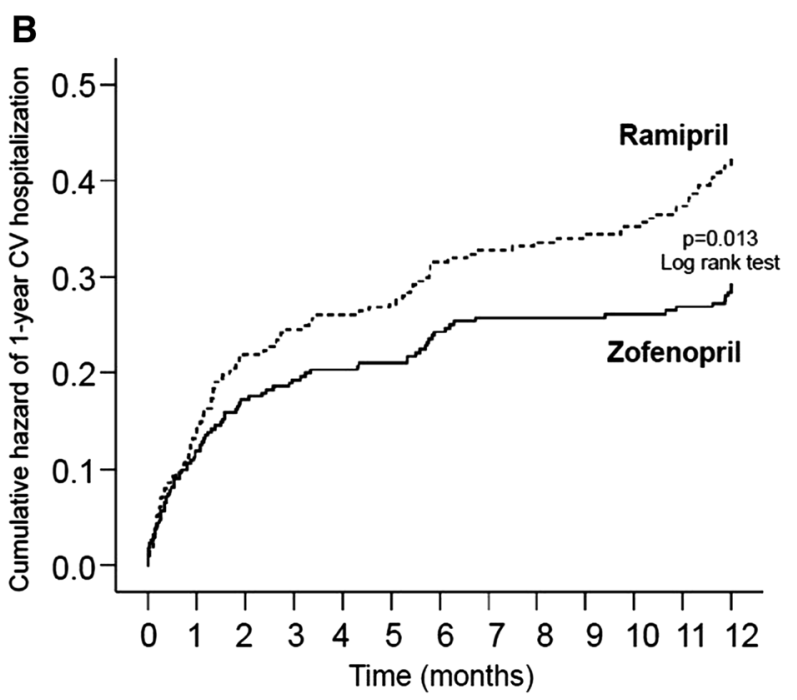

Number at risk

Zofenopril $\quad 365317298287281276267262261260258255247$ Ramipril $\quad 351305276269263260247243238236233228215$

with zofenopril plus ASA (continuous lines, $n=365$ ) or ramipril plus ASA (dashed lines, $n=351$ ) in the SMILE- 4 study. $p$ value from the log rank statistics. $C V$ cardiovascular. Redrawn from Ref. [15] with permission

characteristics, rather than to the clinical impact of therapy [17]. The propensity score was estimated based on 13 variables: age, gender, heart rate, concomitant diabetes, metabolic syndrome, hypercholesterolemia, low highdensity lipoprotein (HDL), type of infarction, revascularization, Killip class, NT-proBNP, LVEF, and glomerular filtration rate. Data on these variables were available for all patients and were considered as representative of the individual's risk level. After adjusting for the propensity score, the superiority of zofenopril over ramipril was confirmed, with overlapping results with those reported in the original SMILE-4 study (OR 0.70, 95\% CI 0.51-0.96, $p=0.028$ ); the rate of major $\mathrm{CV}$ events was similar between propensity groups. However, a significantly lower risk of combined occurrence of death and hospitalization for CV causes was observed in patients in the third quintile of the propensity score, namely in patients who were at intermediate risk (Fig. 2). Based on this observation, subjects at intermediate risk may have more benefit from early treatment with 

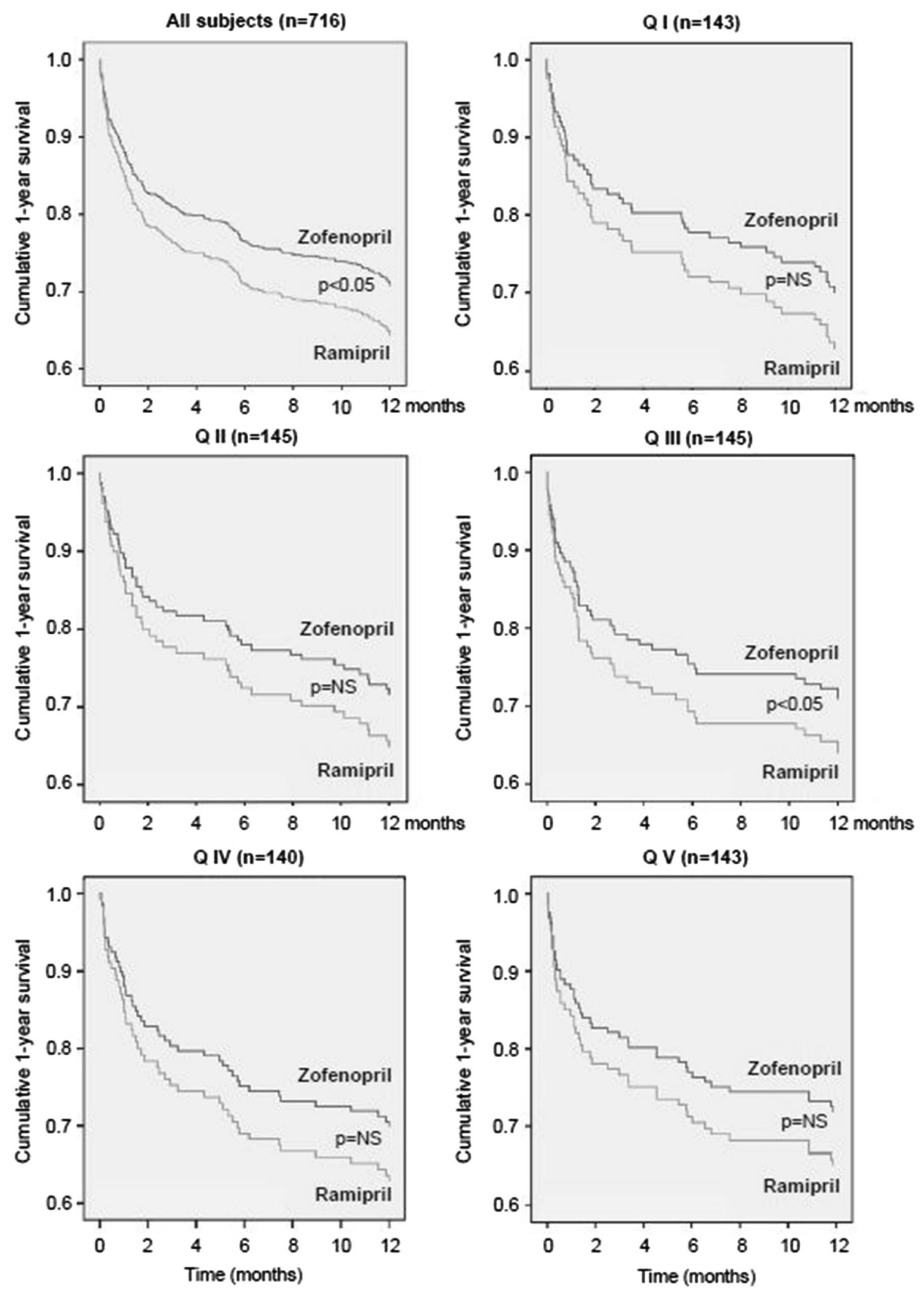
4Fig. 2 Cumulative survival free from CV events between zofenopril and ramipril within quintiles of propensity score at 1 year in the patients of the SMILE- 4 study. The $p$ values in each panel indicate the statistical significance of the between-treatment difference (log rank statistics). $Q$ quintile Redrawn from Ref. [17] with permission

zofenopril, whereas in other risk classes both treatments may be equally effective [17].

\section{COST-EFFECTIVENESS ANALYSIS OF THE SMILE-4 STUDY}

Once clinical efficacy and safety of zofenopril in combination with ASA was demonstrated, a pharmaco-economic analysis was performed to verify whether the added benefit of zofenopril was worth the added cost [18]. Based on the reduction by $30 \%$ of the risk of death or hospitalization for $\mathrm{CV}$ causes with zofenopril compared to ramipril, a number needed to treat of 13 was calculated, thus indicating that one major CV event could be prevented by treating 13 patients fewer with zofenopril than with ramipril. The incremental cost-effectiveness ratio (ICER) for zofenopril compared to ramipril was 2125.45 euros for any additional event prevented. The analysis included costs derived from drug purchase and hospitalization or events (Table 2); the perspective adopted for the study was that of a third-party payer. The information on costs of medications were derived from the national pharmaceutical formulary of each country, while the costs for postAMI events were calculated considering a Diagnosis-Related Group Code [19]. Therefore, the cost-effectiveness of zofenopril was favorable compared to ramipril, a drug already proven to be cost-effective in patients at high risk of CV disease [20], and thus zofenopril could be placed among viable and cost-effective options for managing patients with LVD.

\section{SUBGROUP ANALYSES OF HIGH- RISK PATIENTS OF THE SMILE-4 STUDY}

The wide population of the SMILE-4 Study offers the opportunity to evaluate the efficacy of

Table 2 Unit and overall costs for treating each single event in the SMILE-4 Study From Ref. [18] with permission

\begin{tabular}{|c|c|c|c|c|c|}
\hline \multirow[t]{2}{*}{ Type of outcome } & \multirow{2}{*}{$\begin{array}{l}\text { Cost per event per patient } \\
\text { (euros) }\end{array}$} & \multicolumn{2}{|c|}{ Zofenopril $(n=365)$} & \multicolumn{2}{|c|}{ Ramipril $(n=351)$} \\
\hline & & $\begin{array}{l}\text { No. of } \\
\text { events }\end{array}$ & $\begin{array}{l}\text { Total cost } \\
\text { (euros) }\end{array}$ & $\begin{array}{l}\text { No. of } \\
\text { events }\end{array}$ & $\begin{array}{l}\text { Total cost } \\
\text { (euros) }\end{array}$ \\
\hline Congestive heart failure & 3425.51 & 4 & $13,702.04$ & 7 & $23,978.57$ \\
\hline $\begin{array}{l}\text { Acute myocardial } \\
\text { infarction }\end{array}$ & 4019.04 & 13 & $52,247.52$ & 16 & $64,304.64$ \\
\hline Angina pectoris & 2642.24 & 20 & $52,844.80$ & 22 & $58,129.28$ \\
\hline $\begin{array}{l}\text { Decline in LVEF } \\
\quad>15 \%\end{array}$ & 1141.84 & 15 & $17,127.55$ & 28 & $31,971.43$ \\
\hline Revascularization & $10,993.14$ & 25 & $274,828.38$ & 32 & $351,780.32$ \\
\hline Other causes & 3650.20 & 11 & $40,152.20$ & 12 & $43,802.40$ \\
\hline Death & 4257.64 & 17 & $72,379.88$ & 11 & $46,834.04$ \\
\hline Overall cost & & & $523,282.37$ & & $620,800.68$ \\
\hline Average cost per patient & & & 4983.64 & & 4850.01 \\
\hline
\end{tabular}

Number of occurrences for each outcome are also indicated

$L V E F$ left ventricular ejection fraction 
Table 3 Absolute and relative frequency (\%), adjusted odds ratio (95\% confidence interval) and corresponding $p$ value of 1-year combined occurrence of death or hospitalization for CV causes, of 1-year CV hospitalization and 1-year CV death in subgroups of patients of the SMILE-4 study From Ref. [15] with permission

\begin{tabular}{|c|c|c|c|c|}
\hline & $\begin{array}{l}\text { Zofenopril } \\
n(\%)\end{array}$ & $\begin{array}{l}\text { Ramipril } \\
n(\%)\end{array}$ & $\begin{array}{l}\text { Adjusted odds ratio } \\
\text { ( } 95 \% \text { confidence interval) }\end{array}$ & $p$ value \\
\hline \multicolumn{5}{|l|}{ Arterial hypertension } \\
\hline 1-year combined end-point & $84 / 273(30.8)$ & $99 / 252(39.3)$ & $0.64(0.43,0.96)$ & 0.031 \\
\hline 1-year CV hospitalization & $71 / 260(27.3)$ & $90 / 243(37.0)$ & $0.61(0.40,0.92)$ & 0.017 \\
\hline 1-year CV mortality & $13 / 273(4.8)$ & $9 / 252(3.6)$ & $1.37(0.57,3.30)$ & 0.484 \\
\hline \multicolumn{5}{|l|}{ Diabetes mellitus } \\
\hline 1-year combined end-point & $21 / 68(30.9)$ & $26 / 63(41.3)$ & $0.56(0.18,1.73)$ & 0.313 \\
\hline 1-year CV hospitalization & $16 / 63(25.4)$ & $23 / 60(38.3)$ & $0.38(0.15,0.95)$ & 0.038 \\
\hline 1-year CV mortality & $5 / 68(7.4)$ & $3 / 63(4.8)$ & $8.57(0.57,128.04)$ & 0.120 \\
\hline \multicolumn{5}{|l|}{ Preserved LVEF (> 40\%) } \\
\hline 1-year combined end-point & $48 / 213(22.5)$ & $77 / 235(32.8)$ & $0.59(0.39,0.91)$ & 0.015 \\
\hline 1-year CV hospitalization & $45 / 210(21.4)$ & $74 / 232(31.9)$ & $0.58(0.38,0.89)$ & 0.013 \\
\hline 1-year CV mortality & $3 / 213(1.4)$ & $3 / 235(1.3)$ & $1.11(0.22,5.53)$ & 0.904 \\
\hline
\end{tabular}

$C V$ cardiovascular, $L V E F$ left ventricular ejection fraction

zofenopril in peculiar subgroups of patients, including hypertensive and diabetic subjects and those with preserved ventricular function (Table 3). Before discussing these results obtained in specific subgroups, the post hoc nature of these analyses must be remembered and thus the need of future prospective evaluation of zofenopril versus ramipril in these subgroups taking into account adequate sample sizes and appropriate inclusion/exclusion criteria.

Hypertension is an independent negative prognostic risk factor for AMI and, after AMI, for the incidence of $\mathrm{CV}$ adverse events, likely due to endothelial damage, atherosclerosis, insulin resistance, left ventricular hypertrophy, and ventricular arrhythmias, increases [21]. In the SMILE-4 Study, 525 (77\%) patients were classified as hypertensives [22]. These patients were older, more often females, with higher prevalence of comorbidities, such as diabetes mellitus, hyperlipidemia, angina pectoris, heart failure, and chronic renal failure than normotensives, but were submitted less frequently at entry to PTCA than normotensives. The combined occurrence of death or hospitalization for $\mathrm{CV}$ causes was similar between normotensive and hypertensive patients; however, the effects of zofenopril in reducing the risk of the combined end-point (OR 0.64, 95\% CI $0.43-0.96)$ and the hospitalization for major $\mathrm{CV}$ events (OR 0.61, 95\% CI 0.40-0.92) compared to ramipril were significantly higher in hypertensive patients $(p=0.031$ for the combined end-point and $p=0.017$ for mortality; Table 3). The superiority of zofenopril was particularly striking in the high-risk subgroup of patients with isolated systolic hypertension (OR 0.48, 95\% CI 0.23-0.99, $p=0.045$; Fig. 3). Such effects of zofenopril may be prevalently related to ancillary properties of the drug on the remodelling process and endothelial dysfunction, rather than to its antihypertensive action because the magnitude of blood pressure reduction of zofenopril and ramipril was similar [22].

Diabetes is another comorbidity highly present in the post-AMI setting which increases the risk of developing LVD and worsens congestive 
HF [23, 24]. Zofenopril shows favorable features that may confer potential advantages in patients with AMI complicated by congestive HF. A post hoc analysis of the SMILE-1 study indicated that the efficacy of an early treatment with zofenopril was higher in diabetic patients than in non-diabetic ones, in terms of the reduction of the combined incidence of death and severe congestive $\mathrm{HF}$, with decreased incidence of congestive $\mathrm{HF}$ alone and improved early survival rate [25]. In the SMILE-4 study, in which diabetic patients represented $19 \%$ of the population, zofenopril had a larger effect than ramipril in reducing the combined occurrence of death or hospitalization in patients with diabetes (OR 0.56, 95\% CI 0.18-1.73), although the between-treatment difference was not statically significant $(p=0.313)$. Conversely, the reduction in the rate of hospital admission for $\mathrm{CV}$ causes was significantly larger under zofenopril (OR 0.38, 95\% CI 0.15-0.95, $p=0.038$ ) (Table 3) [26]. The larger effect of zofenopril over ramipril was particularly evident in patients with uncontrolled blood glucose $(\geq 126 \mathrm{mg} / \mathrm{dL})$, regardless of the previous diagnosis of diabetes (Fig. 4). However, in diabetic patients, no superiority of zofenopril was observed in terms of risk reduction of CV mortality (Table 3; Fig. 4). These results are remarkable because the association between diabetes,

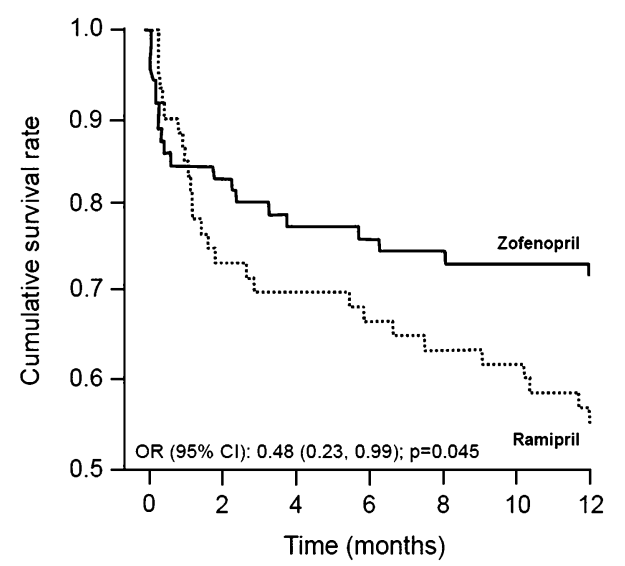

Fig. 3 Cumulative survival without events during 1-year in patients of the SMILE-4 study with isolated systolic hypertension treated with zofenopril (continuous line) or ramipril (dotted line). $p$ value from the logistic regression analysis. $O R$ odds ratio, $C I$ confidence interval Redrawn from Ref. [22] with permission glucose abnormalities, or impaired glucose intolerance and poor outcomes is well documented [27, 28]; zofenopril may contribute to prevent major adverse cardiovascular events in diabetic patients post-AMI who are at further risk.

In the SMILE-4 Study, $63 \%$ of patients had a LVEF > 40\% [15, 29]: data from this population may be useful in the controversy about the use of ACE-inhibitors in patients with preserved LVEF, even if current guidelines consider it as reasonable $[2,3]$. During the study, all patients underwent a standard echocardiographic examination, with determination of LVEF and left ventricular systolic and diastolic volumes, as recommended by the American Society of Echocardiography [30]. Patients with preserved LVEF had a lower prevalence of anterior infarct, higher frequency of PTCA at the admission, higher levels of glomerular filtration rate, and lower values of NT-proBNP and heart rate $[15,29]$. In these patients, zofenopril confirmed its superiority over ramipril with a reduction of combined death and hospitalization for $\mathrm{CV}$

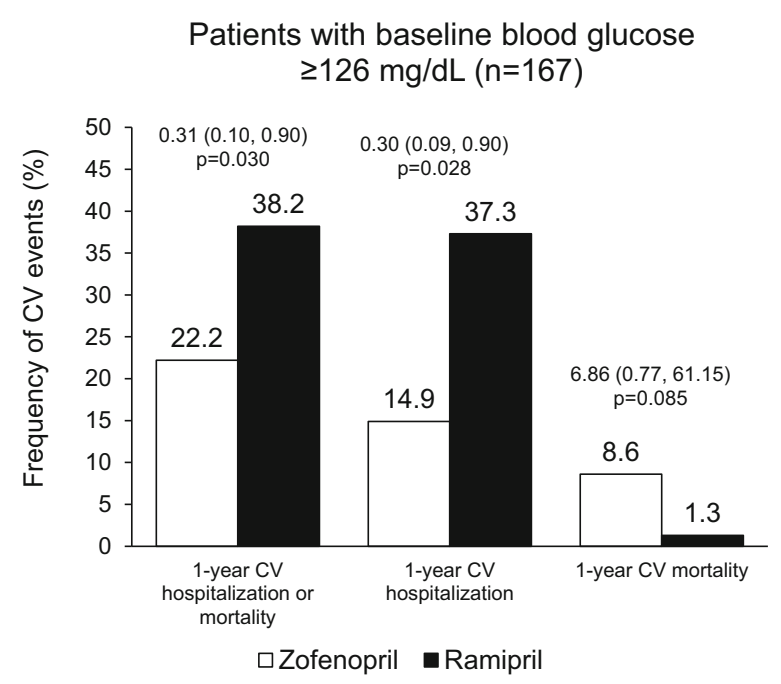

Fig. 4 Prevalence (\%) of 1-year combined occurrence of death or hospitalization for $\mathrm{CV}$ causes, of 1-year $\mathrm{CV}$ hospitalization and 1-year CV death in patients of the SMILE-4 study with elevated baseline blood glucose ( $\geq 126 \mathrm{mg} / \mathrm{dL}$ ) regardless of the presence or absence of diabetes, treated with zofenopril (open bars) or ramipril (full bars). Adjusted odds ratio and 95\% confidence intervals, and $p$ values are shown. $C V$ cardiovascular Redrawn from Ref. [26] with permission 

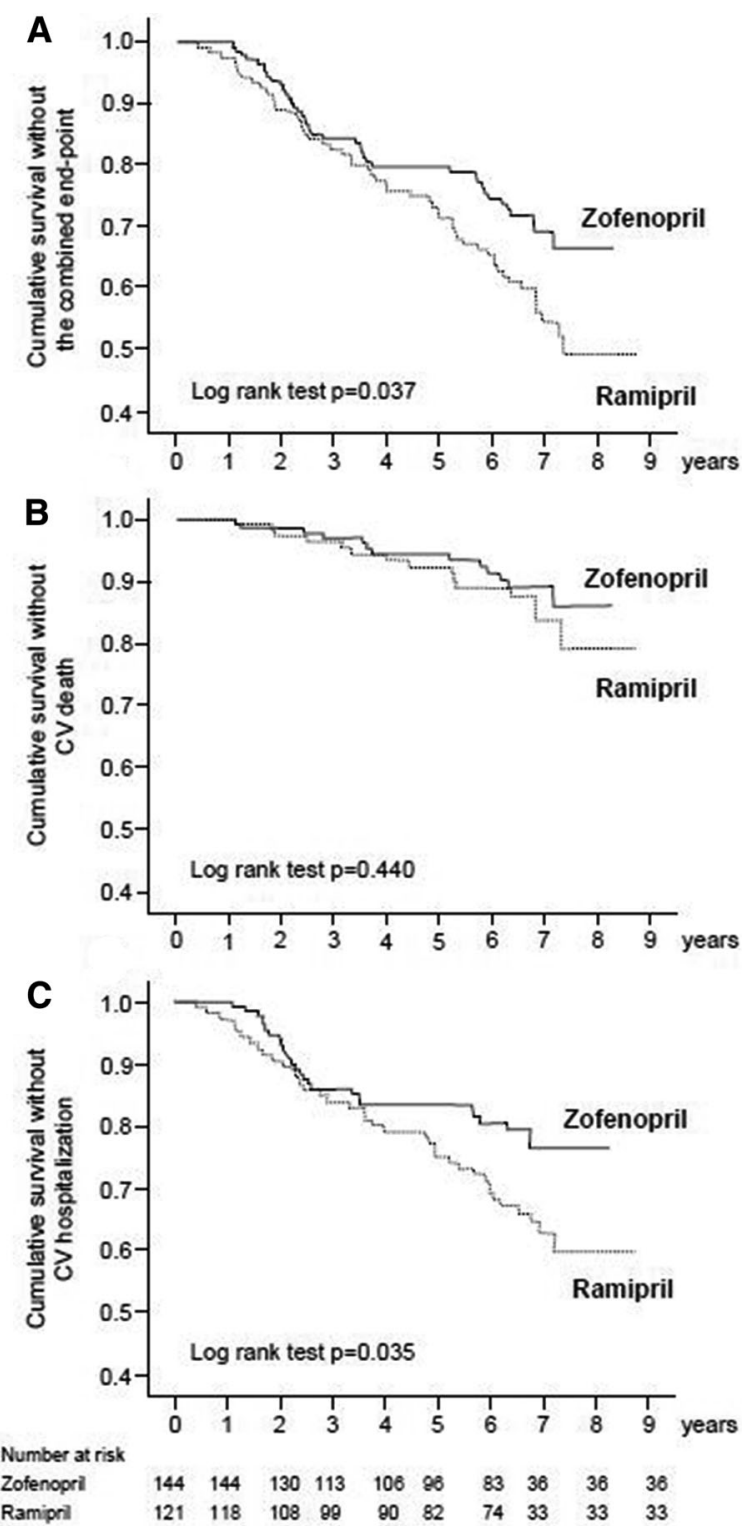

Fig. 5 Incidence of $\mathrm{CV}$ mortality or hospitalization for $\mathrm{CV}$ causes (a), CV death (b) or CV hospitalization (c) during the follow-up in patients originally randomized and treated with zofenopril (continuous lines, $n=144$ ) or ramipril (dashed lines, $n=121$ ) in the SMILE- 4 study. $p$ value from the log rank statistics. $C V$ cardiovascular. Redrawn from [31] with permission

causes by $41 \%$ (OR $0.59,95 \%$ CI $0.39-0.91$, $p=0.015$ ) (Table 3). Consistently with previous results, the reduction in the hospital rate drove the difference between treatments, but the number of deaths in the preserved LVEF group was too small (3 patients in each arm) to provide a conclusive evidence (Table 3 ). Therefore, the efficacy of zofenopril in patients with HF without ECG signs of left ventricular dysfunction may differ from other ACE-inhibitors and the number of patients needed to treat to prevent additional death may be different in patients with preserved or impaired LVEF.

\section{LONG-TERM EFFICACY OF ZOFENOPRIL AND RAMIPRIL IN POST-AMI PATIENTS: THE SMILE- 4 FOLLOW-UP}

The SMILE-4 Study lasted from March 2005 to July 2009 [15]; in 2012, the investigators designed a further follow-up phase to investigate the long-term benefits of early treatment after AMI with zofenopril + ASA or ramipril + ASA [31]. The survival analysis started on the date of entry into the original study; therefore, a median follow-up of 5.5 years was considered. During this time, $28.9 \%$ of patients maintained the originally assigned treatment, $34.9 \%$ switched to the other study drug, and $36.2 \%$ used other ACE-inhibitors, different from zofenopril or ramipril. Furthermore, during the maintenance therapy, dosing was likely different from the initial $60 \mathrm{mg}$ of zofenopril and $10 \mathrm{mg}$ of ramipril. Both switching and different dosing represented confounding factors to determine the occurrence of $\mathrm{CV}$ death or hospitalization during the follow-up; however, on the other hand, these changes strengthened the role on long-term outcomes of early treatment after AMI. Zofenopril maintained its superiority in reducing the combined occurrence of death or hospitalization for $\mathrm{CV}$ causes over ramipril $(27.8 \%$ vs. $43.8 \%)$ and in increasing the survival likelihood after AMI (OR 0.65, 95\% CI $0.43-0.98, p=0.041$ ) (Fig. 5). The rate of hospital admission was significantly lower with zofenopril (OR 0.61, 95\% CI 0.37-0.99, $p=0.047$ ). Interestingly, as shown in Fig. 6, which reports unpublished data from the SMILE-4 follow-up, long-term event-free survival was better in patients originally randomized to zofenopril and continuing the originally assigned treatment or originally treated with 

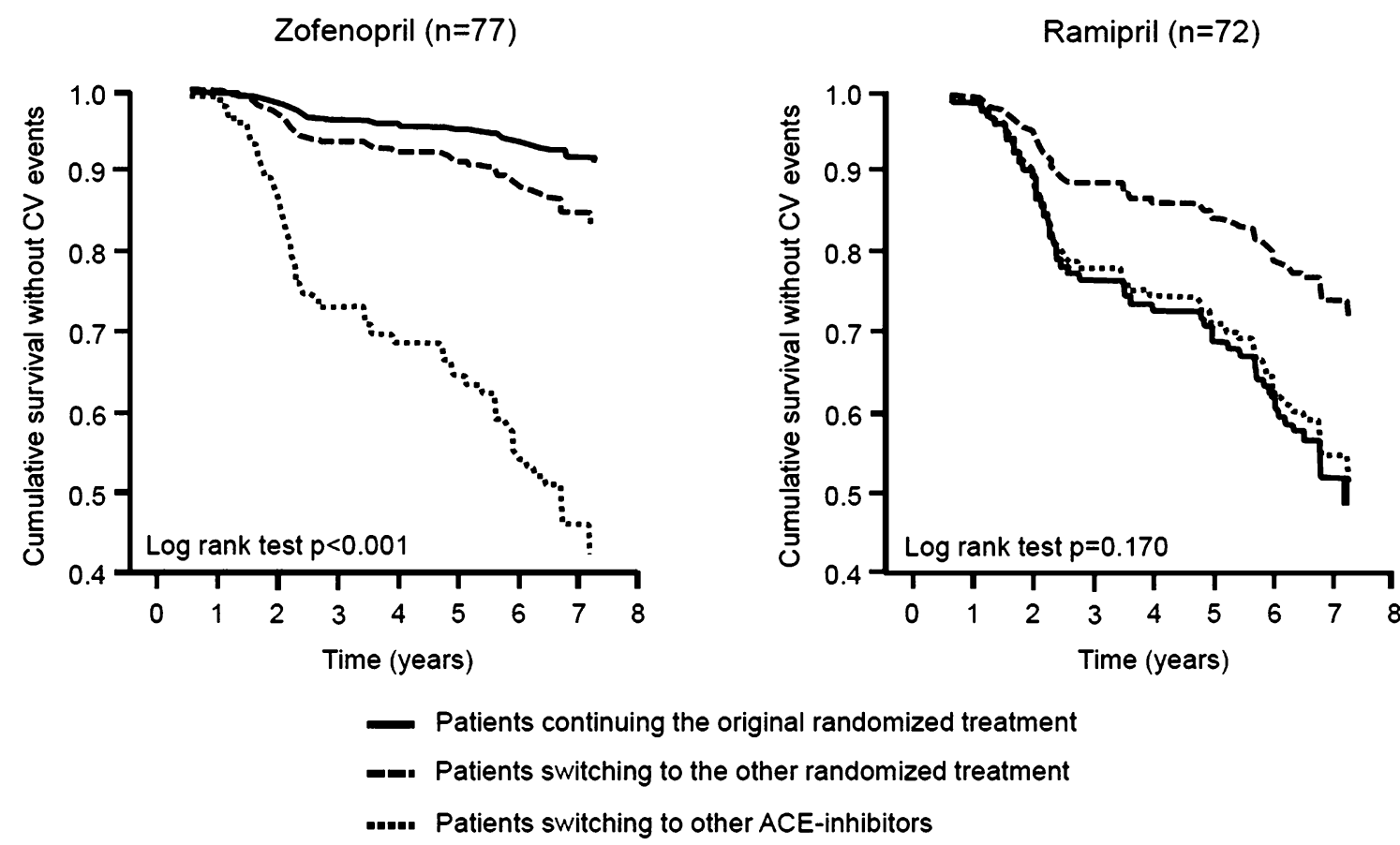

Fig. 6 Cumulative survival without $\mathrm{CV}$ events in the SMILE-4 follow-up. Data are separately shown for patients originally randomized and treated with zofenopril or ramipril, and continuing the original randomized treatment

ramipril and switched to zofenopril in the course of the years than in patients continuing treatment with ramipril. These follow-up data indicated that an early treatment with zofenopril after AMI offered more favorable long-term effects than that with ramipril and confirmed that a combination with ASA did not affect the clinical outcomes of ACE inhibitors.

\section{EFFECTS OF ZOFENOPRIL AND RAMIPRIL ON MORTALITY IN PATIENTS WITH CHRONIC HF IN THE CLINICAL PRACTICE SETTING}

Pharmacoepidemiologic data from real-world settings have indicated that ramipril was associated with better outcomes on HF prognosis, compared to enalapril and captopril [32]. Based on SMILE-4 post-AMI results of zofenopril and ramipril, these two drugs were head-to-head- (continuous line), switching to the other randomized treatment (dashed line) or switching to other ACEinhibitors (dotted line). $p$ values from the log rank statistics. $C V$ cardiovascular, $A C E$ angiotensin converting enzyme

tested in patients with chronic HF to evaluate the outcomes in mortality in clinical practice [33]. In the overall population, mortality was similar among patients who received zofenopril (45 deaths from 102 patients) or ramipril (48 deaths from 73 patients), but zofenopril was a significant predictor of survival in patients older than 76 years (OR 0.56 , 95\% CI 0.35-0.91), in men (OR 0.57, 95\% CI 0.30-0.98), and in patients with ejection fraction lower than $54 \%$ (OR 0.52, 95\% CI 0.26-0.97) (Fig. 7). These subcategories of patients were those, a priori, with a greater probability of readmittance to hospital because of HF [34], and those with greater costs for their management [35].

\section{CONCLUSION}

Early treatment with zofenopril after AMI shows major clinical benefits in patients with LVD compared with ramipril: the effects are sustained over time, with a reduction of 

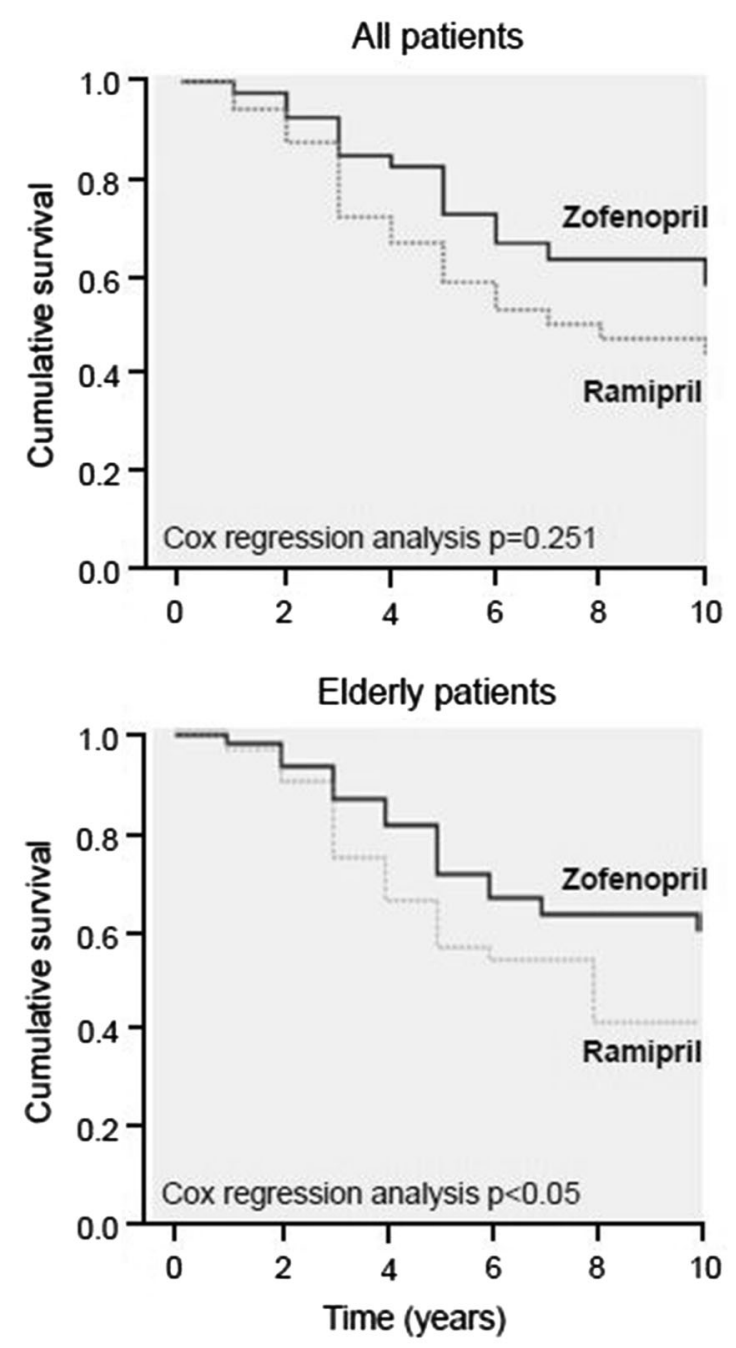

Fig. 7 Cumulative survival in heart failure patients according to treatment with zofenopril (continuous line) or ramipril (dotted line). Data are shown for the whole

hospitalization for $\mathrm{CV}$ cause and death for at least 5 years, and the therapy is cost-effective. The combination with ASA does not seem to affect the antihypertensive activity of zofenopril and its ancillary properties, mainly associated with cardiac and vascular protection. Overall, the results of the SMILE-4 study highlight the superiority of zofenopril over ramipril in combination with ASA, at least in terms of the prevention of cardiovascular hospitalization, and support the early use of zofenopril immediately after AMI. The efficacy of zofenopril was also good in the presence of comorbidities, such as hypertension and diabetes, and

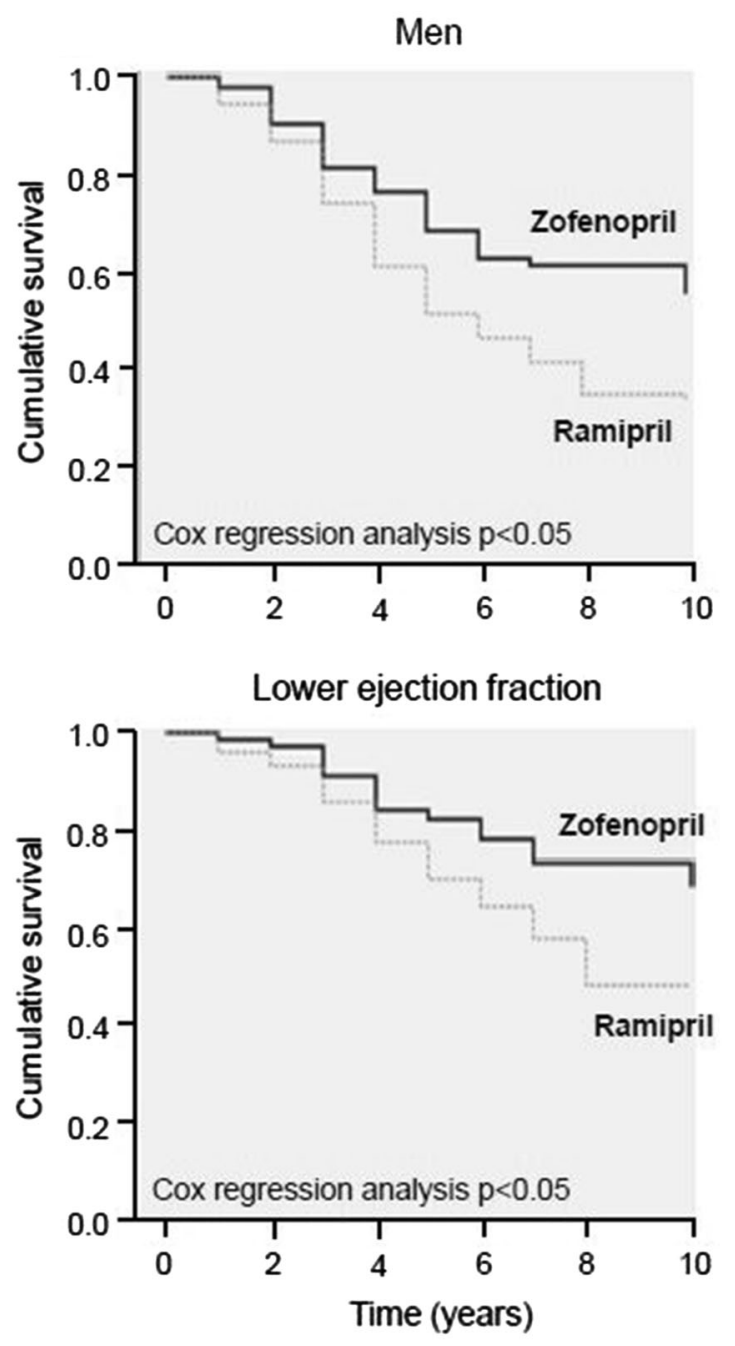

study sample and according to male gender, old age $(>76$ years) and low left ventricular ejection fraction $(<54 \%)$ Redrawn from Ref. [33] with permission

in case of preserved ventricular function, though its superiority versus ramipril was only marginal and not always statistically significant, as in the whole study population. In the postAMI setting, zofenopril should be maintained and may be useful to reduce the risk of $\mathrm{HF}$, especially in men and elderly patients.

\section{ACKNOWLEDGEMENTS}

Funding. Sponsorship for the SMILE programme was funded by Menarini International 
Operations Luxembourg S.A. No funding or sponsorship was received for the publication of this article.

Authorship. All named authors meet the International Committee of Medical Journal Editors (ICMJE) criteria for authorship for this article, take responsibility for the integrity of the work as a whole, and have given their approval for this version to be published. All authors had full access to all of the data in this study and take complete responsibility for the integrity of the data and accuracy of the data analysis.

Disclosures. Conflict of interest of Prof Claudio Borghi: consultancy for Boheringer Ingelheim, Menarini International, Sanofi, Amgen, Takeda, Novartis, Ely Lilly and Servier. Stefano Omboni, Salvatore Novo, Dragos Vinereanu, Giuseppe Ambrosio and Ettore Ambrosioni have nothing to disclose.

Compliance with Ethics Guidelines. This article is based on previously conducted studies and does not contain any new studies with human participants or animals performed by any of the authors.

Open Access. This article is distributed under the terms of the Creative Commons Attribution-NonCommercial 4.0 International License (http://creativecommons.org/licenses/ by-nc/4.0/), which permits any noncommercial use, distribution, and reproduction in any medium, provided you give appropriate credit to the original author(s) and the source, provide a link to the Creative Commons license, and indicate if changes were made.

\section{REFERENCES}

1. Ma TK, Kam KK, Yan BP, Lam YY. Renin-angiotensin-aldosterone system blockade for cardiovascular diseases: current status. Br J Pharmacol. 2010;160:1273-92.

2. Fihn SD, Gardin JM, Abrams J, American College of Cardiology Foundation/American Heart Association Task Force, et al. 2012 ACCF/AHA/ACP/AATS/
PCNA/SCAI/STS guideline for the diagnosis and management of patients with stable ischemic heart disease: a report of the American College of Cardiology Foundation/American Heart Association task force on practice guidelines, and the American College of Physicians, American Association for Thoracic Surgery, Preventive Cardiovascular Nurses Association, Society for Cardiovascular Angiography and Interventions, and Society of Thoracic Surgeons. Circulation. 2012;2012(126):e354-471.

3. Anderson JL, Adams CD, Antman EM, American College of Cardiology Foundation/American Heart Association Task Force on Practice Guidelines, et al. 2012 ACCF/AHA focused update incorporated into the ACCF/AHA 2007 guidelines for the management of patients with unstable angina/non-ST-elevation myocardial infarction: a report of the American College of Cardiology Foundation/ American Heart Association Task Force on Practice Guidelines. Circulation. 2012;2013(127):e663-828.

4. Subissi A, Evangelista S, Giachetti A. Preclinical profile of zofenopril: an angiotensin converting enzyme inhibitor with peculiar cardioprotective properties. Cardiovasc Drug Rev. 1999;17:115-33.

5. Ranadive SA, Chen AX, Serajuddin AT. Relative lipophilicities and structural-pharmacological considerations of various angiotensin-converting enzyme (ACE) inhibitors. Pharm Res. 1992;9:1480-6.

6. Del Corso F, Pareo I, Borghi C. Evolving concept of cardioprotection in myocardial infarction: from SMILE-1 to SMILE-5. Kardiol Pol. 2013;71(7):667-74.

7. Napoli C, Sica V, de Nigris F, et al. Sulfhydryl angiotensin-converting enzyme inhibition induces sustained reduction of systemic oxidative stress and improves the nitric oxide pathway in patients with essential hypertension. Am Heart J. 2004;148(1):e5.

8. Bucci M, Vellecco V, Cantalupo A, et al. Hydrogen sulfide accounts for the peripheral vascular effects of zofenopril independently of ACE inhibition. Cardiovasc Res. 2014;102:138-47.

9. Napoli C, Cicala C, D'Armiento FP, et al. Beneficial effects of ACE-inhibition with zofenopril on plaque formation and low-density lipoprotein oxidation in watanabe heritable hyperlipidemic rabbits. Gen Pharmacol. 1999;33(6):467-77.

10. Carnicelli V, Frascarelli S, Zucchi R. Effect of acute and chronic zofenopril administration on cardiac gene expression. Mol Cell Biochem. 2011;352(1-2):301-7.

11. Donnarumma E, Ali MJ, Rushing AM, et al. Zofenopril protects against myocardial ischemia- 
reperfusion injury by increasing nitric oxide and hydrogen sulfide bioavailability. J Am Heart Assoc. 2016;5(7):e003531.

12. Ambrosioni E, Borghi C, Magnani B. The effect of the angiotensin-converting-enzyme inhibitor zofenopril on mortality and morbidity after anterior myocardial infarction. The Survival of Myocardial Infarction Long-term Evaluation (SMILE) Study Investigators. $\mathrm{N}$ Engl J Med. 1995;332(2):80-5.

13. Borghi C, Ambrosioni E, Survival of Myocardial Infarction Long-term Evaluation-2 Working Party. Double-blind comparison between zofenopril and lisinopril in patients with acute myocardial infarction: results of the Survival of Myocardial Infarction Long-term Evaluation-2 (SMILE-2) study. Am Heart J. 2003;145(1):80-7.

14. Borghi C, Ambrosioni E, Survival of Myocardial Infarction Long-term Evaluation Study Group. Effects of zofenopril on myocardial ischemia in post-myocardial infarction patients with preserved left ventricular function: the Survival of Myocardial Infarction Long-term Evaluation (SMILE)-ISCHEMIA study. Am Heart J. 2007;153:445.e7-14.

15. Borghi C, Ambrosioni E, Novo S, Vinereanu D, Ambrosio G, SMILE-4 Working Party. Comparison between zofenopril and ramipril in combination with acetylsalicylic acid in patients with left ventricular systolic dysfunction after acute myocardial infarction: results of a randomized, double-blind, parallel-group, multicenter, European study (SMILE-4). Clin Cardiol. 2012;35(7):416-23.

16. Borghi C, Omboni S, Reggiardo G, Bacchelli S, Degli Esposti D, Ambrosioni E. Cardioprotective role of zofenopril in patients with acute myocardial infarction: a pooled individual data analysis of four randomised, double-blind, controlled, prospective studies. Open Heart. 2015;2(1):e000220.

17. Borghi C, Omboni S, Novo S, Vinereanu D, Ambrosio G, Ambrosioni E, SMILE-4 Working Party. Zofenopril and ramipril in patients with left ventricular systolic dysfunction after acute myocardial infarction: a propensity analysis of the Survival of Myocardial Infarction Long-term Evaluation (SMILE) 4 study. J Renin Angiotensin Aldosterone Syst 2016;17(4):1470320316656480.

18. Borghi C, Ambrosioni E, Omboni S, et al. D. Costeffectiveness of zofenopril in patients with left ventricular systolic dysfunction after acute myocardial infarction: a post hoc analysis of SMILE4. Clinicoecon Outcomes Res. 2013;5:317-325.

19. Diagnosis-Related Groups in Europe. Towards efficiency and quality. http://www.eurodrg.eu/. Accessed 13 Feb 2014.
20. Malik IS, Bhatia VK, Kooner JS. Cost effectiveness of ramipril treatment for cardiovascular risk reduction. Heart. 2001;85:539-43.

21. Richards AM, Nicholls MG, Troughton RW, et al. Antecedent hypertension and heart failure after myocardial infarction. J Am Coll Cardiol. 2002;39:1182-8.

22. Borghi C, Ambrosioni E, Omboni S, SMILE-4 Working Party, et al. Zofenopril and ramipril and acetylsalicylic acid in postmyocardial infarction patients with left ventricular systolic dysfunction: a retrospective analysis in hypertensive patients of the SMILE-4 study. J Hypertens. 2013;31:1256-64.

23. Bartnik M, Ryden L, Ferrari R, et al. The prevalence of abnormal glucose regulation in patients with coronary artery disease across Europe. Eur Heart J. 2004;25:1880-90.

24. Ovbiagele B, Markovic D, Fonarow CG. Recent US patterns and predictors of prevalent diabetes among acute myocardial infarction patients. Cardiol Res Pract. 2011;2011:145615.

25. Borghi C, Bacchelli S, Degli Esposti D, Ambrosioni $\mathrm{E}$, on behalf of the SMILE Study Investigators. Effects of the early ACE inhibition in diabetic nonthrombolyzed patients with anterior acute myocardial infarction. Diabetes Care. 2003;26:1862-8.

26. Borghi C, Omboni S, Novo S, Vinereanu D, Ambrosio G, Ambrosioni E, SMILE-4 Working Party. Zofenopril and ramipril in combination with Acetyl Salicylic Acid in postmyocardial infarction patients with left ventricular systolic dysfunction: a retrospective analysis of the SMILE-4 randomized, double-blind study in diabetic patients. Cardiovasc Ther. 2016;34:76-84.

27. Hofsten DE, Logstrup BB, Moller JE, Pellika PA, Egstrup K. Abnormal glucose metabolism in acute myocardial infarction. JACC Cardiovasc Imaging. 2009;2:592-9.

28. Mazurek M, Kowalczyk J, Lenarczyk R, et al. The prognostic value of different glucose abnormalities in patients with acute myocardial infarction treated invasively. Cardiovasc Diabetol. 2012;11:78.

29. Borghi C, Omboni S, Cicero AF, et al. Randomised comparison of zofenopril and ramipril plus acetylsalicylic acid in postmyocardial infarction patients with left ventricular systolic dysfunction: a post hoc analysis of the SMILE-4 Study in patients according to levels of left ventricular ejection fraction at entry. Open Heart. 2015;2(1):e000195.

30. Lang RM, Bierig M, Devereux RB, Chamber Quantification Writing Group, American Society of 
Echocardiography's Guidelines and Standards Committee, European Association of Echocardiography, et al. Recommendations for chamber quantification: a report from the American Society of Echocardiography's Guidelines and Standards Committee and the Chamber Quantification Writing Group, developed in conjunction with the European Association of Echocardiography, a branch of the European Society of Cardiology. J Am Soc Echocardiogr. 2005;18:1440-63.

31. Borghi C, Omboni S, Novo S, Vinereanu D, Ambrosio G, Ambrosioni E. Early treatment with zofenopril and ramipril in combination with acetyl salicylic acid in patients with left ventricular systolic dysfunction after acute myocardial infarction: results of a 5-year follow-up of patients of the Smile4 Study. J Cardiovasc Pharmacol. 2017;69(5):298-304.
32. Pilote L, Abrahamowicz M, Eisenberg M, Humphries $\mathrm{K}$, Behlouli $\mathrm{H}$, Tu JV. Effect of different angiotensin-converting-enzyme inhibitors on mortality among elderly patients with congestive heart failure. Can Med Assoc J. 2008;178:1303-11.

33. Borghi C, Cosentino ER, Rinaldi ER, Cicero AF. Effect of zofenopril and ramipril on cardiovascular mortality in patients with chronic heart failure. Am J Cardiol. 2013;112(1):90-3.

34. Coffey RM, Misra A, Barrett M, Andrews RM, Mutter R, Moy E. Congestive heart failure: who is likely to be readmitted? Med Care Res Rev. 2012;69:602-16.

35. Norton C, Georgiopoulou VV, Kalogeropoulos AP, Butler J. Epidemiology and cost of advanced heart failure. Prog Cardiovasc Dis. 2011;54:78-85. 